\title{
Representation of Image Data by New Lifting based Biorthogonal Wavelets and Its Application to Lossless Compression
}

\author{
M. Santhosh ${ }^{1}$, B. Stephen Charles ${ }^{2}$, Dr. M N Giriprasad ${ }^{3}$ \\ ${ }^{1}$ Asso. Prof., Dept. of ECE, SSCET, Kurnool, INDIA \\ ${ }^{2}$ Principal, SSCET, Kurnool, INDIA \\ ${ }^{3} H O D$, Dept. of ECE, JNTUA, Anantapuramu, INDIA
}

\begin{abstract}
In this paper an attempt has been made to derive the lifting scheme for a set of new bi-orthogonal wavelets and apply on image compression. Four new bi-orthogonal wavelets are designed by taking different basis functions which are selected so as to capture the sharp edges which are common in images. For these classical wavelets, lifting versions are calculated and presented in this paper. The lifting step calculation is simplest among the schemes in related literature. From the designed wavelet filters, the poly-phase matrix is written and decomposed the matrix into a form from which lifting steps are directly available. To incorporate coding Set Partitioning In Hierarchical Trees (SPIHT) algorithm is used. The Peak Signal to Noise Ratio (PSNR), Compression Ratio (CR), Transforming Time (TT), Encoding Time (ET) and Decoding Times (DT) are calculated. It was found that the compression performance of new classical wavelets is a little better than that of existing classicalffirst generation wavelets and that of new lifting based wavelets is far better than that of existing classical wavelets and also existing lifting based wavelets.
\end{abstract}

Keywords: Lifting scheme, basis functions, compression, poly-phase representation

\section{INTRODUCTION}

Lifting Scheme which was first introduced by Sweldens is the tool to construct wavelet in spatial domain entirely. It is the key technology to build second generation wavelet. Lifting Scheme can implement wavelet transform which can't be translated and dilated from one function. Moreover, it can lead to a fast, fully in-place implementation of wavelet transform[1][2]. This algorithm is faster, simpler and easier than the Mallat algorithm. It's also the one recommended by JPEG2000 for its good performance. All parameters of Lifting Scheme can be modified, but not affect the properties of perfect reconstruction after transform. The benefit of using lift scheme for wavelet transform is that it decomposes the wavelet filter into simple step, and each step is reversible. The reconstruct process is the reversed decomposition in lifting. The classical wavelet transforms are linear, and their constructions are often based on the Fourier transform. However, it is changed after Sweldens[1] proposed the lifting scheme. The lifting scheme is a flexible tool for construction of new wavelets from existing ones. A general lifting scheme, illustrated in Figure 1, comprises three main steps: split, predict and update.

The three steps form a lifting stage. Daubechies and Sweldens [3] have shown that all classical wavelets decompositions can be implemented using the lifting scheme. The predict and update operators can reverse the order [4]. It is very useful of the reversion of the order sometimes when the update happens before predict. Many people proposed various kinds of nonlinear wavelets at the basis of lifting scheme [5][6]. However, in all these approaches, a severe limitation is that the filter structure is fixed, and thus cannot cope with the sudden changes in the input signal.

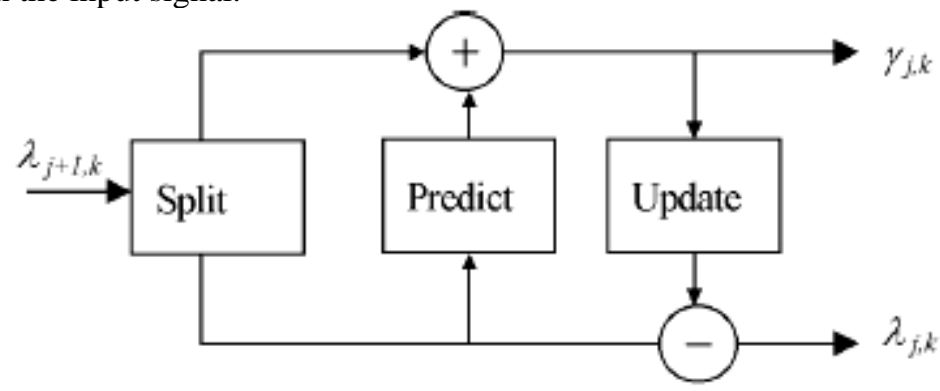

Fig. 1 Lifting Scheme

Since more than a decade, a number of papers on lifting, on its variations and extensions are published. In [7], a modified lifting scheme for computing the approximation and detailed coefficients of DWT is proposed. The modified equations use, right shift operators and 6-bit multipliers. 
The hierarchy levels in computation are reduced to one; thereby minimizing the delay and increasing throughput. In [8], efficient, and simple design, of multilevel two dimensional discrete wavelet transform (2-D DWT) modules for image compression was presented. The proposed architecture is based on lifting scheme approach, using the (5/3) wavelet filter, aiming to reduce the hardware complexity and size of the on-chip memory. In [9], a high speed lifting based 3D (DWT) VLSI architecture is proposed. The architecture was arranged in efficient way to speed up and achieve higher hardware utilization. In [10], a framework for constructing adaptive wavelet decompositions using the lifting scheme was proposed. A major requirement is that perfect reconstruction is possible with better quality. Once coefficients are generated, the best directional window sizes are determined to obtain the best reconstructed image, which can be considered as an optimization task. In [11], an algorithm for color medical image compression based on a biorthogonal wavelet transform CDF 9/7 coupled with SPIHT coding algorithm was proposed.

In [12], Bi-orthogonal wavelets are constructed using lifting scheme that makes optimal use of both high pass and low pass filter values, and consequently addition and shift operations are performed on the resulting wavelet coefficients. It projects the advantages of IWT using LS used in real time compression of both still and color images involving non smooth domains or curves. In this paper, the wavelets proposed in [13] are considered, and for these wavelets the lifting scheme is calculated and so generated lifting wavelets are applied on image for compression. Because of the selection of the basis functions for these wavelets, the performance is found to be the best among the wavelets in literature. The rest of the paper is organized as follows. The next section presents lifting scheme and signal representation with lifting scheme. It also presents the poly-phase representation of DWT and lifting scheme. The section III presents the calculation of lifting steps for the new biorthogonal wavelets. The section IV gives the simulation results of proposed wavelets and a brief review of the performance of different wavelets in literature.

\section{LIFTING SCHEME}

Let us consider the sequence of samples of signal $\mathrm{x}(\mathrm{k}) . \mathrm{Z}$ transform of this sequence can be given as,

$$
X(z)=\sum_{k} x(k) z^{-k}
$$

Let us consider finite impulse response (FIR) filter $h$ having filter coefficients $h=\{h k 1, \ldots$, hk 2$\}$. $Z$ transform of this filter is Laurent polynomial with degree $|\mathrm{k} 2-\mathrm{k} 1|$ given by,

$$
H(z)=\sum_{k=k 1}^{k=k 2} h_{k} z^{-k}
$$

Filtering of signal $\mathrm{x}(\mathrm{k})$ by filter $\mathrm{h}$ can be easily described in $\mathrm{z}$ transform by the (3)

$$
\mathrm{Y}(\mathrm{z})=\mathrm{H}(\mathrm{z}) \mathrm{X}(\mathrm{z})
$$

Sub-sampling of the signal $x(k)$ is corresponding to keeping only the even samples i.e. $x e=x(2 k)$. $Z$ transform of such sub-sampled signal can be given as

$$
\begin{gathered}
X_{e}(z)=\sum_{k} x(2 k) z^{-k} \\
\mathrm{X}(\mathrm{z})=\mathrm{x}(0) \mathrm{z}^{0}+\mathrm{x}(1) \mathrm{z}^{1}+\mathrm{x}(2) \mathrm{z}^{2}+\mathrm{x}(3) \mathrm{z}^{3}+\ldots \ldots \\
\mathrm{X}(-\mathrm{z})=\mathrm{x}(0) \mathrm{z}^{0}-\mathrm{x}(1) \mathrm{z}^{1}+\mathrm{x}(2) \mathrm{z}^{2}-\mathrm{x}(3) \mathrm{z}^{3}+\ldots \ldots \\
\mathrm{X}_{\mathrm{e}}\left(\mathrm{z}^{2}\right)=1 / 2[\mathrm{X}(\mathrm{z})+\mathrm{X}(-\mathrm{Z})]=\sum_{k} x(2 k) z^{-2 k}
\end{gathered}
$$

Similarly

$$
\mathrm{X}_{\mathrm{o}}\left(\mathrm{z}^{2}\right)=(\mathrm{z} / 2)[\mathrm{X}(\mathrm{z})-\mathrm{X}(-\mathrm{Z})]=\sum_{k} x(2 k+1) z^{-2 k}
$$

From (5) and (6), it is clear that the signal $X(z)$ can be decomposed into $X_{e}\left(z^{2}\right)$ and $X_{o}\left(z^{2}\right)$ as given in (7).

$$
\mathrm{X}(\mathrm{z})=\mathrm{X}_{\mathrm{e}}\left(\mathrm{z}^{2}\right)+\mathrm{z}^{-1} \mathrm{X}_{\mathrm{o}}\left(\mathrm{z}^{2}\right)
$$

Now let us consider that signal $\mathrm{X}(\mathrm{z})$ decomposed into two parts using high pass filter $\mathrm{g}$ and low pass filter $\mathrm{h}$, then it can be represented as:

$$
\left[\begin{array}{l}
\operatorname{lp}(z) \\
h p(z)
\end{array}\right]=\left[\begin{array}{c}
H(z) \\
G(z)
\end{array}\right] X(z)
$$

Sub-sampling step corresponds to

$$
L P\left(z^{2}\right)=\operatorname{lp}_{e}\left(z^{2}\right)=\frac{\operatorname{lp}(z)+\operatorname{lp}(-z)}{2}=\frac{H(z) X(z)+H(-z) X(-z)}{2}
$$




$$
H P\left(z^{2}\right)=l p_{o}\left(z^{2}\right)=\frac{h p(z)+h p(-z)}{2}=\frac{G(z) X(z)+G(-z) X(-z)}{2}
$$

The above equations can be written in matrix form as

$$
\left[\begin{array}{l}
L P\left(z^{2}\right) \\
H P\left(z^{2}\right)
\end{array}\right]=\left[\begin{array}{l}
l p_{e}\left(z^{2}\right) \\
l p_{o}\left(z^{2}\right)
\end{array}\right]=\frac{1}{2}\left[\begin{array}{cc}
H(-z) & H(z) \\
G(-z) & G(z)
\end{array}\right]\left[\begin{array}{c}
X(-z) \\
X(z)
\end{array}\right]
$$

In this case we first calculate all the coefficients and then throw away half of the work done. It will be more efficient if we perform sampling before filtering, mean that we compute only even part of $\mathrm{lp}$ and $\mathrm{hp}$.

Similarly,

$$
l p_{e}(z)=[H(z) X(z)]_{e}=H_{e}(z) X_{e}(z)+z^{-1} H_{o}(z) X_{o}(z)
$$

$$
h p_{e}(z)=[G(z) X(z)]_{e}=G_{e}(z) X_{e}(z)+z^{-1} G_{o}(z) X_{o}(z)
$$

Let us denote output of sub-sampler and low pass filter as $\lambda(z)$ and output of sub-sampler and high pass filter as $\gamma(\mathrm{z})$. Then above two equations can be represented as,

$$
\left[\begin{array}{l}
\lambda(z) \\
\gamma(z)
\end{array}\right]=P(z)\left[\begin{array}{c}
X_{e}(z) \\
z^{-1} X_{0}(z)
\end{array}\right]
$$

Where, $\mathrm{P}(\mathrm{z})$ is a poly-phase matrix is given by

$$
P(z)=\left[\begin{array}{ll}
H_{e}(z) & H_{o}(z) \\
G_{e}(z) & G_{o}(z)
\end{array}\right]
$$

In order to achieve perfect reconstruction, filter $\mathrm{h}$ and $\mathrm{g}$ must be complementary filters that will result unity determinant of poly-phase matrix. Polyphase matrix corresponding to lazy wavelet transform will be

$$
P(z)=\left[\begin{array}{ll}
1 & 0 \\
0 & 1
\end{array}\right]
$$

This poly-phase matrix will split input samples into odd and even set.

\section{CALCUlation OF LifTING STEPS FOR NEW WAVELETS}

In this section the lifting steps for the new orthogonal wavelets for which the basis functions are shown in figure 2. In the first wavelet the decomposition functions are somewhat similar to that of Haar. But the value during non-zero period is not constant; it is decaying from 1 to 0.9 . The reconstruction function is smooth and it is a sinusoidal variation. In the second wavelet, the same reconstruction stage as it is in first wavelet is used. The decomposition stage is a sharp and short wave that is supposed to capture dissimilar neighbourhood values in the input data. The wavelet filters for these wavelets are calculated and for simplicity let $\mathrm{h}=\left\{\mathrm{h}_{-4}, \mathrm{~h}_{-3}, \mathrm{~h}_{-2}, \mathrm{~h}_{-1}, \mathrm{~h}_{0}, \mathrm{~h}_{1}, \mathrm{~h}_{2}, \mathrm{~h}_{3}, \mathrm{~h}_{4}\right\}$ and $\ell=\left\{\ell_{-4}, \ell_{-3}, \ell_{-2}, \ell_{-1}, \ell_{0}, \ell_{1}, \ell_{2}, \ell_{3}, \ell_{4}\right\}$

For the proposed wavelets $h_{-4}=0, \ell_{-4}=0$.

The poly-phase matrix before the circular convolution $P^{\prime}(z)=\left[\begin{array}{cc}H_{e}^{\prime}(z) & H^{\prime}{ }_{o}(z) \\ \ell_{e}^{\prime}(z) & \ell^{\prime}{ }_{o}(z)\end{array}\right]$

$$
\therefore \quad P^{\prime}(z)=\left[\begin{array}{ll}
h_{-2} z^{2}+h_{0}+h_{2} z^{-2}+h_{4} z^{-4} & h_{-3} z^{3}+h_{-1} z+h_{1} z^{-1}+h_{3} z^{-3} \\
\ell_{-2} z^{2}+\ell_{0}+\ell_{2} z^{-2}+\ell_{4} z^{-4} & \ell_{-3} z^{3}+\ell_{-1} z+\ell_{1} z^{-1}+\ell_{3} z^{-3}
\end{array}\right]
$$



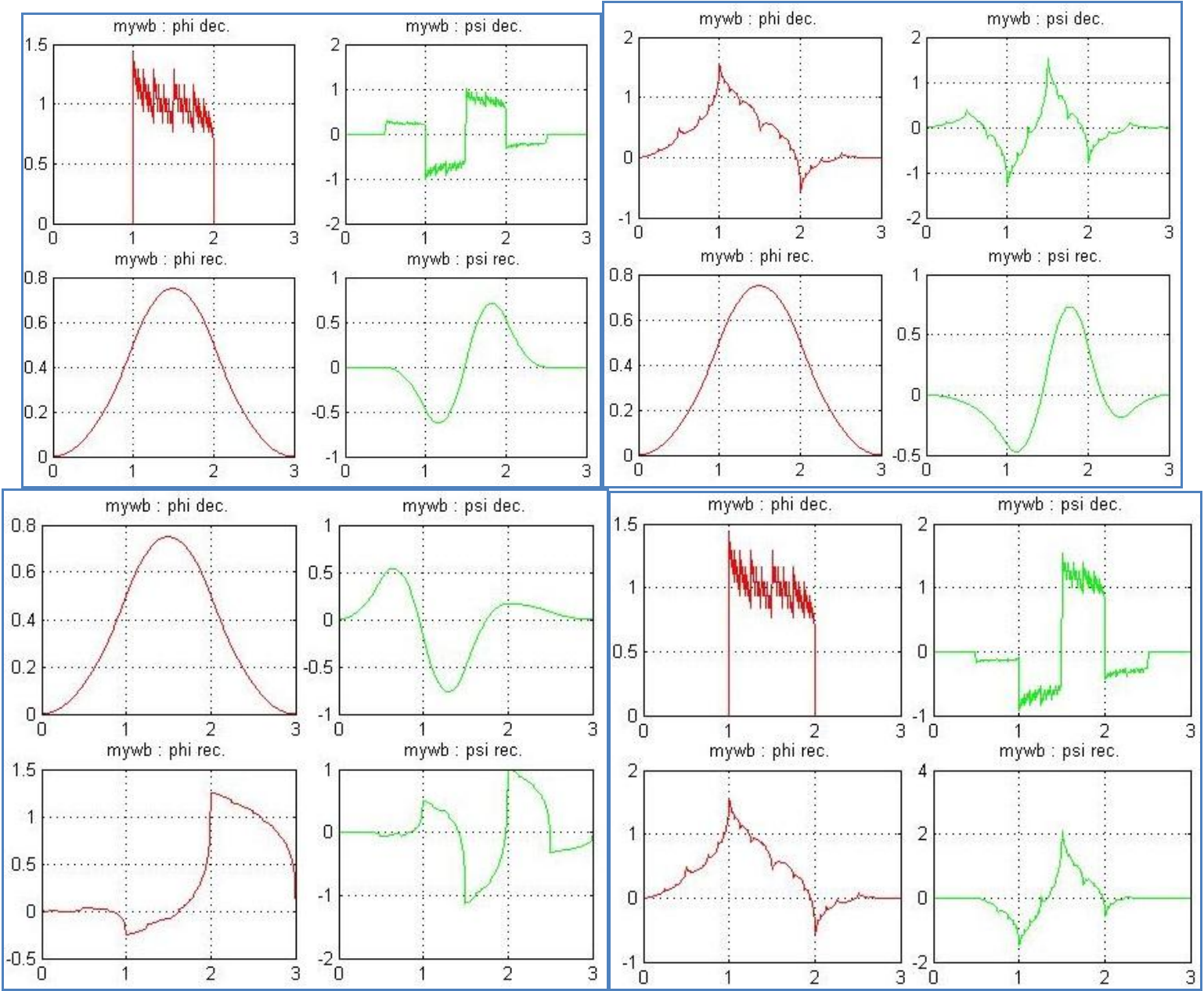

Fig. 2 New Wavelets: phi and psi functions of NBior1, NBior2, NBior3 and NBior4

We obtain the Poly-phase matrix P(z) by applying circular convolution to the elements of P'(z). Therefore

$$
\begin{aligned}
P(z) & =\left[\begin{array}{ll}
h_{-2} z+h_{0}+h_{2} z^{-1}+h_{4} z^{-2} & h_{-3} z^{2}+h_{-1} z+h_{1}+h_{3} z^{-1} \\
\ell_{-2} z+\ell_{0}+\ell_{2} z^{-1}+\ell_{4} z^{-2} & \ell_{-3} z^{2}+\ell_{-1} z+\ell_{1}+\ell_{3} z^{-1}
\end{array}\right] \\
& =\left[\begin{array}{cc}
H_{e}(z) & H_{o}(z) \\
\ell_{e}(z) & \ell_{o}(z)
\end{array}\right]
\end{aligned}
$$

Now $\mathrm{P}(\mathrm{z})$ can be decomposed into two matrices as

From equations (17) and (18),

$$
P(z)=\left[\begin{array}{cc}
H_{e}(z) & H_{o}^{N e w}(z) \\
\ell_{e}(z) & \ell_{o}{ }^{N e w}(z)
\end{array}\right]\left[\begin{array}{cc}
1 & S(z) \\
0 & 1
\end{array}\right]
$$

$$
\begin{aligned}
& H_{o}(z)=H_{0}{ }^{N e w}(z)+s(z) H_{e}(z) \\
& \ell_{o}(z)=\ell_{0}^{N e w}(z)+s(z) \ell_{e}(z)
\end{aligned}
$$

One can obtain $\mathrm{S}(\mathrm{z})$ and $H_{0}{ }^{\text {New }}(z)$ by dividing $\mathrm{H}_{0}(\mathrm{z})$ by $\mathrm{H}_{\mathrm{e}}(\mathrm{z}) \mathrm{S}(\mathrm{z})$ will be the quotient and $H_{0}^{\text {New }}(z)$ will be the remainder. Similarly $\ell_{0}{ }^{N e w}(z)$ will be the remainder in the division $\ell_{0}(\mathrm{z})$ by $\ell_{\mathrm{e}}(\mathrm{z})$.

$\mathrm{S}(\mathrm{z}), H_{0}^{\mathrm{New}}(z)$ and $\ell_{0}{ }^{\mathrm{New}}(z)$ can calculated and given by 


$$
\begin{aligned}
& S(z)=C_{1} z^{-1}+C_{2} z^{-2}+C_{3} z^{-3} \\
& H_{o}^{N e w}(z)=C_{4}+C_{5} z^{-1}+C_{6} z^{-2} \\
& \ell_{o}^{N e w}(z)=C_{7}+C_{8} z^{-1}+C_{9} z^{-2}
\end{aligned}
$$

Where the $\mathrm{C}_{1}, \mathrm{C}_{2}, \mathrm{C}_{3}, \ldots, \mathrm{C}_{9}$ are constants in terms of wavelet filter coefficients. The equation (18) becomes,

$$
P(z)=\left[\begin{array}{cc}
H_{e}(z) & C_{4}+C_{5} z^{-1}+C_{6} z^{-2} \\
\ell_{e}(z) & C_{7}+C_{8} z^{-1}+C_{9} z^{-2}
\end{array}\right]\left[\begin{array}{cc}
1 & C_{1} z^{-1}+C_{2} z^{-2}+C_{3} z^{-3} \\
0 & 1
\end{array}\right]
$$

Now the first matrix in the above equation can further be decomposed into two matrices as,

From equation (22),

$$
\left[\begin{array}{cc}
H_{e}(z) & H_{o}{ }^{N e w}(z) \\
\ell_{e}(z) & \ell_{o}^{N e w}(z)
\end{array}\right]=\left[\begin{array}{cc}
H^{\text {New }}(z) & H_{o}{ }^{N e w}(z) \\
\ell_{e}^{N e w}(z) & \ell_{o}^{\text {New }}(z)
\end{array}\right]\left[\begin{array}{cc}
1 & 0 \\
T(z) & 1
\end{array}\right]
$$

$$
\begin{aligned}
& H_{e}(z)=H_{e}{ }^{N e w}(z)+T(z) H_{o}{ }^{N e w}(z) \\
& \ell_{e}(z)=\ell_{e}^{N e w}(z)+T(z) \ell_{o}^{N e w}(z)
\end{aligned}
$$

As we have calculated S(z), $\quad H_{0}^{\text {New }}(z)$ and $\ell_{0}^{\text {New }}(z)$ we can calculate $\mathrm{T}(\mathrm{z}), H_{e}{ }^{\text {New }}(z)$ and $\ell_{e}^{\text {New }}(z)$ by performing divisions. The $\mathrm{T}(\mathrm{z}), H_{e}^{\text {New }}(z)$ and $\ell_{e}^{\text {New }}(z)$ values are given by,

$$
\begin{aligned}
& T(z)=A_{1} z+A_{2}+A_{3} z^{-1} \\
& H_{e}^{\text {New }}(z)=A_{4}+A_{5} z^{-1} \\
& \ell_{e}^{\text {New }}(z)=A_{6}+A_{7} z^{-1}
\end{aligned}
$$

Now the equation (22) becomes

$$
\left[\begin{array}{cc}
H_{e}(z) & H_{o}^{N e w}(z) \\
\ell_{e}(z) & \ell_{o}^{N e w}(z)
\end{array}\right]=\left[\begin{array}{cc}
A_{4}+A_{5} z^{-1} & H_{o}{ }^{N e w}(z) \\
A_{6}+A_{7} z^{-1} & \ell_{o}^{N e w}(z)
\end{array}\right]\left[\begin{array}{cc}
1 & 0 \\
A_{1} z+A_{2}+A_{3} z^{-1} & 1
\end{array}\right]
$$

By using the equations (21), (22) and (25) the poly-phase matrix $\mathrm{P}(\mathrm{z})$ can be written in a form, so that the lifting steps both primal and dual lifting steps can be calculated.

$$
P(z)=\left[\begin{array}{cc}
A_{4}+A_{5} z^{-1} & H_{o}{ }^{N e w}(z) \\
A_{6}+A_{7} z^{-1} & \ell_{o}{ }^{N e w}(z)
\end{array}\right]\left[\begin{array}{cc}
1 & 0 \\
A_{1} z+A_{2}+A_{3} z^{-1} & 1
\end{array}\right]\left[\begin{array}{cc}
1 & C_{1} z^{-1}+C_{2} z^{-2}+C_{3} z^{-3} \\
0 & 1
\end{array}\right]
$$

For the wavelet NBior1, the wavelet coefficients, the constants A1 to A9 and C1 to C7 are listed in the table 1,2 and 3 respectively.

TABLE I: Wavelet Coefficients of the Wavelet NBior1

\begin{tabular}{|c|c|}
\hline $\mathbf{h}(\mathbf{n})$ & $\ell(\mathbf{n})$ \\
\hline 0 & 0 \\
\hline 0.1426 & 0.0532 \\
\hline 0.8244 & 0.0761 \\
\hline-0.0929 & 0.2275 \\
\hline-0.4693 & -0.1043 \\
\hline 0.1043 & -0.4693 \\
\hline 0.2275 & 0.0929 \\
\hline-0.0761 & 0.8244 \\
\hline 0.0532 & -0.1426 \\
\hline
\end{tabular}

TABLE II: The Constants C1 to C9 of the Wavelet NBior1

\begin{tabular}{|l|c|}
\hline C1 & 0.1729 \\
\hline C2 & -0.3224 \\
\hline C3 & -0.506 \\
\hline $\mathbf{C 4}$ & -0.2495 \\
\hline
\end{tabular}




\begin{tabular}{|c|c|}
$\mathbf{C 5}$ & 0.1323 \\
\hline $\mathbf{C 6}$ & 0.0269 \\
\hline $\mathbf{C 7}$ & 0.8262 \\
\hline C8 & 0.001 \\
\hline C9 & -0.0721 \\
\hline
\end{tabular}

TABLE III: The Constants A1 to A7 of the Wavelet NBior1

\begin{tabular}{|c|c|}
\hline $\mathbf{A 1}$ & -3.3046 \\
\hline $\mathbf{A 2}$ & 0.1292 \\
\hline $\mathbf{A 3}$ & -1.2 \\
\hline $\mathbf{A 4}$ & 0.2084 \\
\hline $\mathbf{A 5}$ & 0.0323 \\
\hline $\mathbf{A 6}$ & -0.132 \\
\hline $\mathbf{A 7}$ & -0.0866 \\
\hline
\end{tabular}

Hence the lifting scheme for the wavelet NBior1 can be written as follows.

Forward Wavelet Transform:

Split

Dual Lifting (Predict)

Primal Lifting (Update) $\lambda_{\mathrm{k}} \leftarrow \mathbf{x}(2 \mathrm{k})$

$\gamma_{\mathrm{k}} \leftarrow \mathbf{x}(2 \mathrm{k}+1)$

$\gamma_{\mathrm{k}} \leftarrow \gamma_{\mathrm{k}}+\left[-3.3046 \gamma_{\mathrm{k}-1}+0.1292 \gamma_{\mathrm{k}}-1.2 \gamma_{\mathrm{k}+1}\right]$

$\lambda_{\mathrm{k}} \leftarrow \lambda_{\mathrm{k}}+\left[0.1729 \lambda_{\mathrm{k}+1}-0.3224 \lambda_{\mathrm{k}+2}-0.506 \lambda_{\mathrm{k}+3}\right]$

Inverse Wavelet Transform:

Inverse Primal Lifting (Update)

Inverse Dual Lifting (Predict)

Merge

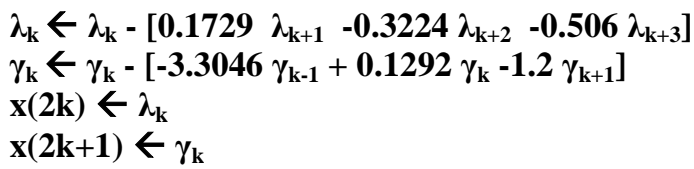

\section{Simulation RESULTS AND DISCUSSION}

In this section, the simulation results of the proposed techniques are presented. The proposed techniques are implemented in MATLAB. The figure 3 shows the GUI used to provide an interface to the user. Before presenting the compression results of the proposed techniques, a mention of the results of existing traditional, new first generation and lifting version of existing traditional wavelets would be appropriate.

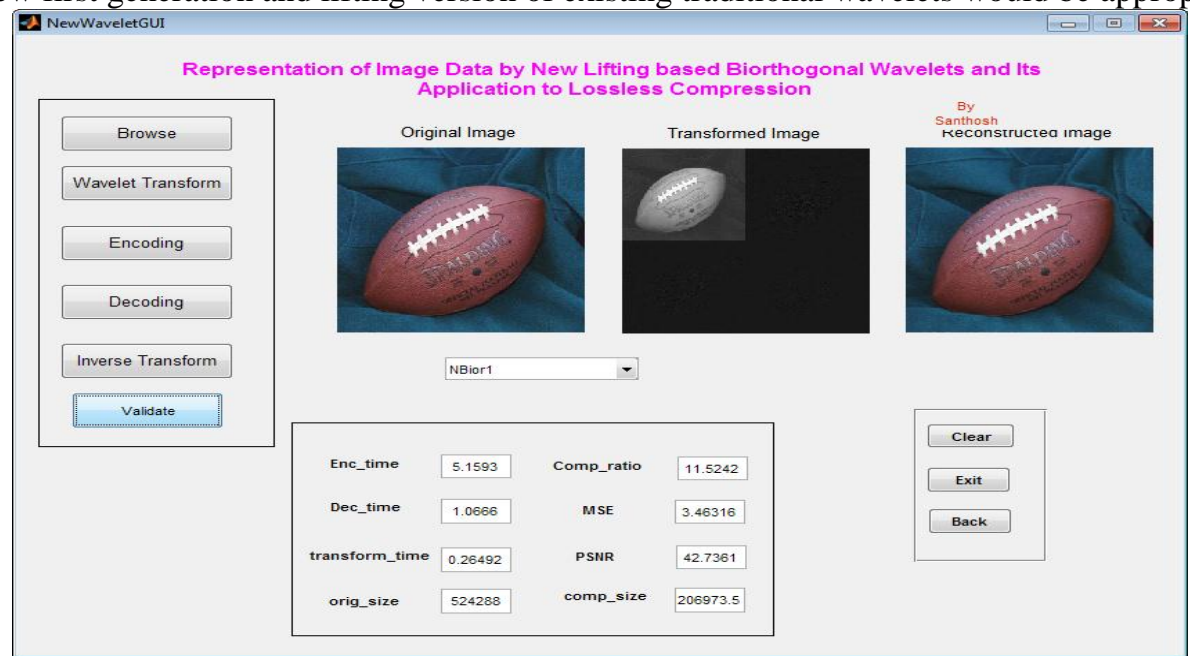

Fig. 3 The GUI used in implementing the proposed Wavelets

The compression results of existing traditional wavelets are given in Tables IV and V. In table IV, the PSNR and CR with four standard images are shown. In table II, the average values of CR, PSNR as well as PSNR*CR are given.

TABLE IV: Compression performance of different wavelets on standard images

\begin{tabular}{|l|l|l|l|l|}
\hline Image & Lena & Mandrill & Pepper & Rice \\
\hline
\end{tabular}




\begin{tabular}{|l|l|l|l|l|l|l|l|l|} 
Wavelet & PSNR & CR & PSNR & CR & PSNR & CR & PSNR & CR \\
\hline Haar & 37.91 & 2.57 & 35.85 & 2.13 & 38 & 2.65 & 38.35 & 2.7 \\
\hline db5 & 38.26 & 2.6 & 35.75 & 2.05 & 38.37 & 2.71 & 38.95 & 2.56 \\
\hline db10 & 36.54 & 2.43 & 31.26 & 1.91 & 36.53 & 2.47 & 37.91 & 2.39 \\
\hline bior1.3 & 33.15 & 2.5 & 28.09 & 2.07 & 31.85 & 2.53 & 34.01 & 2.45 \\
\hline bior2.2 & 35.75 & 2.73 & 29.43 & 2.16 & 36.36 & 2.64 & 38.25 & 2.68 \\
\hline coif1 & 35.19 & 2.62 & 29.14 & 2.1 & 34.3 & 2.73 & 37.35 & 2.57 \\
\hline coif3 & 38.32 & 2.47 & 35.8 & 1.94 & 38.49 & 2.58 & 39.08 & 2.43 \\
\hline sym2 & 30.97 & 2.65 & 27.9 & 2.13 & 29.34 & 2.78 & 29.12 & 2.61 \\
\hline sym3 & 35.51 & 2.65 & 29.52 & 2.11 & 35.35 & 2.77 & 38.06 & 2.61 \\
\hline Dmeyer & 36.21 & 1.46 & 29.72 & 1.12 & 35.92 & 1.46 & 39.08 & 1.43 \\
\hline
\end{tabular}

TABLE V: Average values of PSNR, CR and PSNR *CR

\begin{tabular}{|l|l|l|l|}
\hline Wavelet & PSNR & CR & PSNR ${ }^{*} \mathrm{CR}$ \\
\hline Haar & 37.53 & 2.51 & 94.29 \\
\hline $\mathrm{db} 5$ & 37.83 & 2.48 & 93.82 \\
\hline $\mathrm{db} 10$ & 35.56 & 2.30 & 81.79 \\
\hline bior1.3 & 31.78 & 2.39 & 75.86 \\
\hline bior2.2 & 34.95 & 2.55 & 89.20 \\
\hline coif1 & 34.00 & 2.51 & 85.16 \\
\hline coif3 & 37.92 & 2.36 & 89.31 \\
\hline sym2 & 29.33 & 2.54 & 74.58 \\
\hline sym3 & 34.61 & 2.54 & 87.74 \\
\hline Dmeyer & 35.23 & 1.37 & 48.18 \\
\hline
\end{tabular}

In Tables VI and VII the simulation results of Lifting version of traditional wavelets are presented.

TABLE VI: Performance of Lifting based Wavelets

\begin{tabular}{lcccccccccc}
\hline & Lena & \multicolumn{3}{c}{ Barbara } & \multicolumn{3}{c}{ Mandrill } & \multicolumn{3}{c}{ Cameraman } \\
\hline Lifting Wavelets & CR & PSNR & CR & PSNR & CR & PSNR & CR & PSNR \\
Haar & 3.98 & 32.83 & 3.40 & 31.30 & 3.69 & 25.13 & 2.28 & 37.31 \\
Daubechies & 3.26 & 33.46 & 3.70 & 32.06 & 3.88 & 25.61 & 2.32 & 37.28 \\
Bi-orthogonal & 3.28 & 33.43 & 4.18 & 21.71 & 3.91 & 25.54 & 2.33 & 37.18 \\
CDF & 3.96 & 32.74 & 3.86 & 26.14 & 3.67 & 25.00 & 2.27 & 37.18 \\
Symlet & 3.24 & 33.21 & 3.64 & 31.60 & 3.84 & 25.23 & 2.31 & 36.91 \\
\hline
\end{tabular}

TABLE VII: Average PSNR, CR and PC of Lifting Wavelets

\begin{tabular}{|llll|}
\hline Haar & PSNR & CR & PC \\
\hline Daubechies & 31.64 & 3.34 & 105.62 \\
\hline Biorthogonal & 32.10 & 3.29 & 105.58 \\
\hline CDF & 29.46 & 3.43 & 100.97 \\
\hline Symlet & 30.26 & 3.44 & 104.07 \\
\hline
\end{tabular}

In Table VIII, the simulation results of new traditional wavelets are given. 
TABLE VIII: Average PSNR, CR and PC values

\begin{tabular}{|c|c|c|c|}
\hline & PSNR & CR & PC \\
\hline NBior1 & 33.52 & 3.24 & 108.67 \\
\hline NBior2 & 31.53 & 3.20 & 101.14 \\
\hline NBior3 & 28.56 & 2.95 & 84.45 \\
\hline NBior4 & 32.20 & 2.92 & 94.33 \\
\hline
\end{tabular}

Because of the sharp edge features of the basis functions used in new traditional wavelets, the performance of the new wavelets will be better than the mentioned wavelets. The performance of the lifting version of new wavelets is given in the tables IX to XIX.

TABLE IX: The performance of new lifting based Biorthogonal wavelets on 'cameraman.jpg'

\begin{tabular}{|l|l|l|l|l|}
\hline $\begin{array}{l}\text { Compression results of New Lifting } \\
\text { 'cameraman.jpg' }\end{array}$ & NBiored & NBior2 & NBior3 & NBior4 \\
\hline & NBiorelets with \\
\hline PSNR (dB) & 41.2472 & 40.6959 & 39.6476 & 41.0777 \\
\hline CR (bpp) & 11.7668 & 11.7814 & 11.4025 & 11.3741 \\
\hline TT (sec) & 0.53883 & 0.25545 & 0.25193 & 0.27299 \\
\hline ET $(\mathrm{sec})$ & 5.9006 & 4.9326 & 5.2007 & 5.4897 \\
\hline DT (sec) & 1.4152 & 1.184 & 1.3906 & 1.3235 \\
\hline
\end{tabular}

TABLE X: The performance of new lifting based Biorthogonal wavelets on 'football.jpg'

\begin{tabular}{|l|l|l|l|l|}
\multicolumn{5}{|c|}{ Compression results of New Lifting based Wavelets with } \\
'football.jpg' \\
\hline & NBior1 & NBior2 & NBior3 & NBior4 \\
\hline PSNR (dB) & 42.7361 & 42.3193 & 41.5113 & 43.1308 \\
\hline CR (bpp) & 11.5242 & 11.1549 & 11.1471 & 11.1019 \\
\hline TT (sec) & 0.29303 & 0.45362 & 0.28082 & 0.29622 \\
\hline ET (sec) & 5.406 & 5.7733 & 5.8627 & 5.8602 \\
\hline DT (sec) & 1.0977 & 1.1764 & 2.1292 & 2.2292 \\
\hline
\end{tabular}

TABLE XI: The performance of new lifting based Biorthogonal wavelets on 'greens.jpg'

\begin{tabular}{|l|l|l|l|l|}
\hline \multicolumn{5}{|c|}{ Compression results of New Lifting based Wavelets with } \\
\hline & NBior1 & NBior2 & NBior3 & NBior4 \\
\hline PSNR (dB) & 40.1453 & 39.6106 & 38.5565 & 40.4767 \\
\hline CR (bpp) & 10.9127 & 10.9152 & 10.4133 & 10.3918 \\
\hline TT (sec) & 0.2476 & 0.26175 & 0.24888 & 0.27174 \\
\hline ET (sec) & 6.0507 & 5.9467 & 6.2758 & 6.2551 \\
\hline DT (sec) & 1.6444 & 1.8216 & 2.4544 & 2.4682 \\
\hline
\end{tabular}

TABLE XII: The performance of new lifting based Biorthogonal wavelets on 'lena.jpg'

\begin{tabular}{|l|l|l|l|l|}
\multicolumn{5}{|c|}{ Compression results of New Lifting based Wavelets with } \\
\hline & NBior1 & NBior2 & NBior3 & NBior4 \\
\hline PSNR (dB) & 42.6359 & 42.0561 & 40.9888 & 42.0904 \\
\hline CR (bpp) & 12.4891 & 12.5122 & 11.839 & 11.7392 \\
\hline TT (sec) & 0.27342 & 0.27033 & 0.25686 & 0.25728 \\
\hline ET (sec) & 4.6752 & 4.7516 & 5.4207 & 5.5004 \\
\hline DT (sec) & 0.96943 & 1.0448 & 1.1687 & 1.2546 \\
\hline
\end{tabular}

TABLE XIII: The performance of new lifting based Biorthogonal wavelets on 'pepper.jpg'

\begin{tabular}{|l|l|l|l|l|}
\multicolumn{5}{|c|}{ Compression results of New Lifting based Wavelets with } \\
& NBior1 & NBior2 & NBior3 & NBior4 \\
\hline PSNR (dB) & 42.4042 & 41.6713 & 40.4042 & 41.5272 \\
\hline CR (bpp) & 12.7051 & 12.7077 & 11.9301 & 11.8879 \\
\hline TT (sec) & 0.37603 & 0.26233 & 0.62823 & 0.3107 \\
\hline
\end{tabular}




\begin{tabular}{|l|l|l|l|l|} 
ET (sec) & 4.5708 & 4.7143 & 5.0368 & 4.704 \\
\hline DT $(\mathrm{sec})$ & 1.0727 & 1.1106 & 1.4258 & 1.224 \\
\hline
\end{tabular}

TABLE XIV: The performance of new lifting based Biorthogonal wavelets on 'rice.jpg'

\begin{tabular}{|l|l|l|l|l|}
\multicolumn{5}{|c|}{ Compression results of New Lifting based Wavelets with } \\
\hline & NBior1 & NBior2 & NBior3 & NBior4 \\
\hline PSNR (dB) & 43.0267 & 41.879 & 41.0919 & 42.0703 \\
\hline CR (bpp) & 12.8023 & 12.2914 & 12.1023 & 12.0212 \\
\hline TT (sec) & 0.24464 & 0.24973 & 0.21718 & 0.24305 \\
\hline ET (sec) & 4.3762 & 4.6109 & 4.8759 & 4.7946 \\
\hline DT (sec) & 0.93959 & 0.91315 & 1.1036 & 1.2093 \\
\hline
\end{tabular}

TABLE XV: The performance of new lifting based Biorthogonal wavelets on 'zebra.jpg'

\begin{tabular}{|l|l|l|l|l|}
\hline \multicolumn{5}{|c|}{ Compression results of New Lifting based Wavelets with } \\
'zebra.jpg' \\
\hline & NBior1 & NBior2 & NBior3 & NBior4 \\
\hline PSNR (dB) & 40.855 & 40.1864 & 38.9937 & 40.7237 \\
\hline CR (bpp) & 11.9819 & 11.9869 & 11.0564 & 11.0126 \\
\hline TT $(\mathrm{sec})$ & 0.26054 & 0.25994 & 0.21827 & 0.24726 \\
\hline ET $(\mathrm{sec})$ & 4.8664 & 4.8799 & 5.6967 & 5.5721 \\
\hline DT $(\mathrm{sec})$ & 1.1232 & 1.1507 & 1.5818 & 1.5782 \\
\hline
\end{tabular}

TABLE XVI: The performance of new lifting based Biorthogonal wavelets on 'office_1.jpg'

\begin{tabular}{|l|l|l|l|l|}
\hline \multicolumn{5}{|c|}{ Compression results of New Lifting based Wavelets with } \\
\hline & NBior1 & NBior2 & NBior3 & NBior4 \\
\hline PSNR (dB) & 44.6101 & 44.2936 & 43.4804 & 44.5424 \\
\hline CR (bpp) & 13.802 & 13.8209 & 13.2791 & 13.2769 \\
\hline TT (sec) & 0.25462 & 0.26105 & 0.28212 & 0.26427 \\
\hline ET (sec) & 4.2749 & 4.2233 & 4.565 & 4.6896 \\
\hline DT (sec) & 0.69567 & 0.68591 & 0.78215 & 0.80255 \\
\hline
\end{tabular}

TABLE XVII: The performance of new lifting based Biorthogonal wavelets on 'office_2.jpg'

\begin{tabular}{|l|l|l|l|l|}
\hline \multicolumn{5}{|c|}{ Compression results of New Lifting based Wavelets with 'office_2.jpg' } \\
\hline & NBior1 & NBior2 & NBior3 & NBior4 \\
\hline PSNR (dB) & 38.3515 & 38.3465 & 38.1955 & 38.2572 \\
\hline CR (bpp) & 12.5451 & 12.5532 & 12.0143 & 11.9972 \\
\hline TT (sec) & 0.30019 & 0.25463 & 0.3454 & 0.27155 \\
\hline ET (sec) & 4.4015 & 4.7408 & 4.8468 & 5.0304 \\
\hline DT (sec) & 0.30089 & 0.30664 & 0.31414 & 0.38754 \\
\hline
\end{tabular}

TABLE XVIII: The performance of new lifting based Biorthogonal wavelets on 'office_3.jpg'

\begin{tabular}{|l|l|l|l|l|}
\hline \multicolumn{5}{|c|}{ Compression results of New Lifting based Wavelets with 'office_3.jpg' } \\
\hline & NBior1 & NBior2 & NBior3 & NBior4 \\
\hline PSNR (dB) & 37.8458 & 37.8268 & 37.5818 & 37.7141 \\
\hline CR (bpp) & 12.1069 & 12.1144 & 11.5888 & 11.5751 \\
\hline TT (sec) & 0.24237 & 0.26591 & 0.25372 & 0.27279 \\
\hline ET (sec) & 4.7295 & 4.7836 & 5.1497 & 5.2466 \\
\hline DT (sec) & 0.35337 & 0.40341 & 0.36114 & 0.36395 \\
\hline
\end{tabular}

TABLE XIX: The performance of new lifting based Biorthogonal wavelets on 'office_4.jpg'

\begin{tabular}{|l|l|l|l|l|}
\hline \multicolumn{5}{|c|}{ Compression results of New Lifting based Wavelets with 'office_4.jpg' } \\
\hline & NBior1 & NBior2 & NBior3 & NBior4 \\
\hline PSNR (dB) & 37.827 & 35.7337 & 37.4934 & 37.6594 \\
\hline
\end{tabular}




\begin{tabular}{|l|l|l|l|l|} 
CR (bpp) & 11.9418 & 11.5173 & 11.4252 & 11.4248 \\
\hline TT (sec) & 0.25514 & 0.26339 & 0.30054 & 0.28447 \\
\hline ET (sec) & 5.0341 & 5.3275 & 5.5119 & 5.7407 \\
\hline DT (sec) & 0.4152 & 0.33625 & 0.41154 & 0.44966 \\
\hline
\end{tabular}

The average values of PSNR, CR and PC [15] with the new lifting based wavelets are given the table XX.

TABLE XX: Average values of PSNR, CR and PC with new lifting based wavelets

\begin{tabular}{llll}
\hline & PSNR & CR & PC \\
\hline Nbior1 & 41.15395 & 12.23435 & 503.492 \\
Nbior2 & 40.41993 & 12.12323 & 490.02 \\
Nbior3 & 39.81319 & 11.65437 & 463.9978 \\
Nbior4 & 40.84272 & 11.61843 & 474.5282 \\
\hline
\end{tabular}

The figures 4, 5 and 6 give the comparison of performance of existing traditional, new traditional, lifting version of traditional, 5/3, 9/7 lifting based [14] and lifting version of new biorthogonal wavelets.

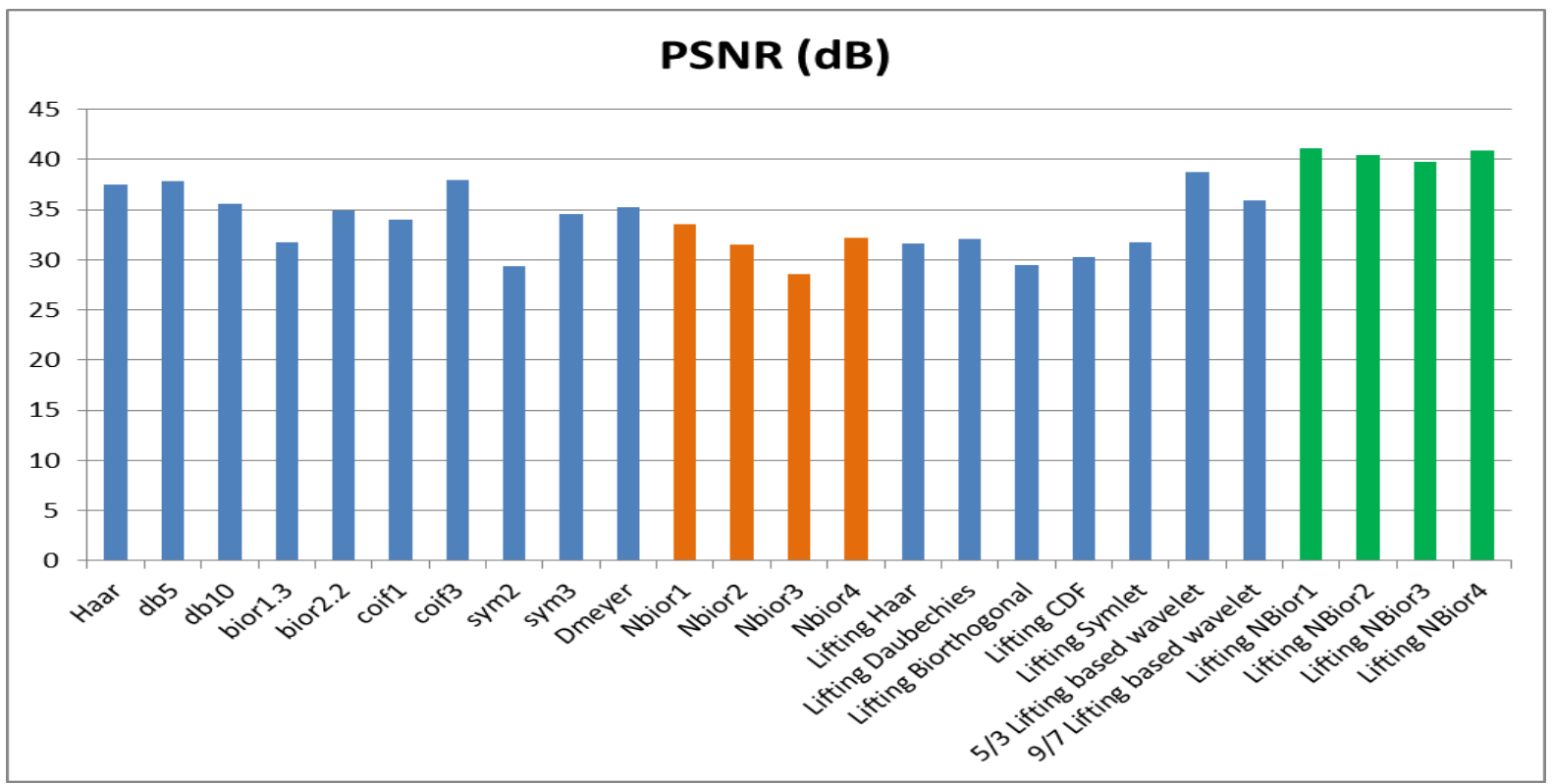

Fig. 4 The PSNR values obtained with various Wavelets 


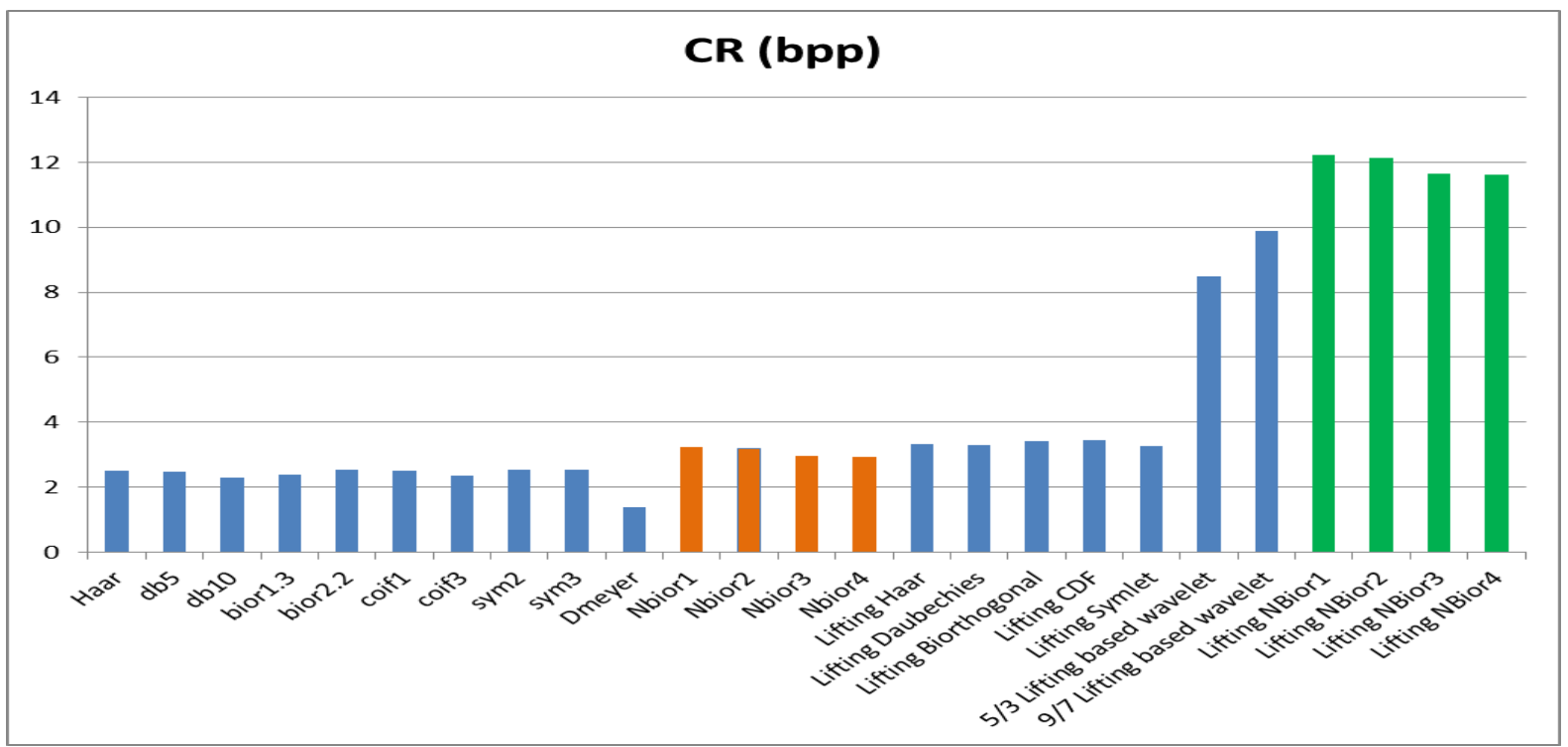

Fig. 5 The CR values obtained with various Wavelets

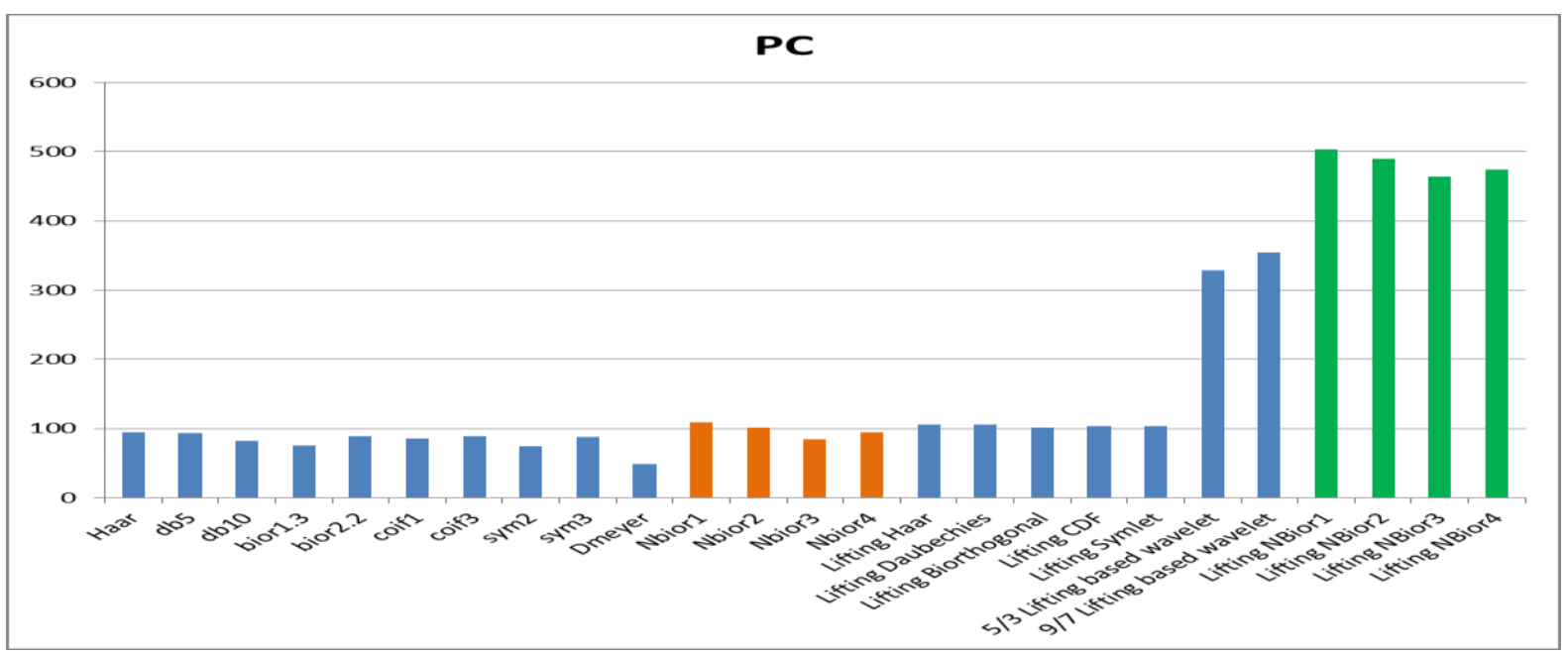

Fig. 6 The PC values obtained with various Wavelets

A number of images are considered to have a subtle comparison of the performance of different wavelets as already mentioned. The PSNR values ranges between 30 to $40 \mathrm{~dB}$. The CR ranges between 1.5 to 12 bpp. The CR with all traditional wavelets is very low, less than 4bpp. With 5/3 and 9/7 lifting based wavelets it between 8 and 10bpp. With the proposed wavelets the CR is over 11bpp.

\section{CONCLUSIONS}

In this paper an attempt has been made to propose a simple procedure to calculate lifting steps for an arbitrary wavelet as well as new set of lifting based wavelets. The procedure presented in this paper is the simplest among the previous and current literature. First of all, new set of functions are taken with short duration and sharp edges. The chosen functions are used as the basis functions for biorthogonal wavelets. By using the lifting step calculation given in the section III, the lifting scheme was facilitated for the new biorthogonal wavelets. These wavelets are used to represent a digital image so as to make things easy for the SPIHT to code the image. The performance of SPIHT is verified with various wavelets, i.e., existing traditional wavelets, new first generation wavelets', lifting version of existing traditional wavelets, 5/3 and $9 / 7$ lifting based wavelet transforms and the new lifting based wavelets, i.e., the lifting version of new first generation wavelets. To compare the performance of all these wavelets, the new design metric PC has been used. Among the existing traditional wavelets haar and db5 has produced the best performance for which the PC is just below 95 . With the new first generation wavelets the PC has crossed 100 and it is 108.67 for NBior1, 101.14 for NBior2. The lifting version of existing traditional wavelets has shown a similar performance. Lifting version of haar produced 105.62 PC, that of daubechies a 105.58, biorthogonal 100.97, CDF 104.07, and Symlet 103.44. The popular 5/3 lifting based wavelet transform has produced a PC of 328.277, and the 9/7 lifting based wavelet transform 
354.52. The new lifting based wavelets proposed in this paper have produced even higher PC value with NBior1 touching 500 .

\section{REFERENCES}

[1] Wim Sweldens. The lifting scheme:A construction of second generation wavelets. SIAMJ. Math. Anal, 29 (1997) 2511-546

[2] Wim Sweldens. The lifting scheme:A custom-design construction of biorthogonal wavelets. Journal of Appl. and Comput. Harmonic Analysis, 3, 2(1996) 186-200.

[3] Daubechies, I., and Sweldens, W. "Factoring wavelet transforms into lifting steps". Journal of Fourier Analysis and Applications 4, 3 (1998), 245-267.

[4] Claypoole, R. L., Davis, G., Sweldens, W., and Baraniuk, R. D." Nonlinear wavelet transforms for image coding". In Proceedings of the 31st Asilomar Conference on Signals, Systems, and Computers, Volume 1 (1997), pp. 662-667.

[5] Heijmans, H. J. A. M., and Goutsias, J. "Nonlinear multiresolution signal decomposition schemes: Part II: morphological wavelets". IEEE Transactions on Image Processing 9,11 (2000), 1897-1913.

[6] Hampson, F. J., and Pesquet, J.-C." A nonlinear subband decomposition with perfect reconstruction". In Proceedings of the IEEE International Conference on Acoustics, Speech, and Signal Processing (Atlanta, Georgia, May 7-10, 1996), pp. 1523-1526.

[7] M. Nagabushanam, S. Ramachandran, P.Kumar, "FPGA Implementation of 1D and 2D DWT Architecture using Modified Lifting Scheme", WSEAS TRANSACTIONS on SIGNAL PROCESSING, Issue 4, Volume 9, October 2013.

[8] Naseer M. Basheer, Mustafa Mushtak Mohammed, "Design and FPGA Implementation of a Lifting Scheme 2D DWT Architecture", International Journal of Recent Technology and Engineering (IJRTE), Volume-2, Issue-1, March 2013.

[9] Senthilkumar.M, Uma.S, "High Speed 3d DWT VISI Architecture for Image Processing Using Lifting Based Wavelet Transform", International Journal of Communication and Computer Technologies Volume 01 - No.36, Issue: 05 May 2013.

[10] R.Ramanathan,K.Kalaiarasi,D.Prabha, "Improved wavelet based compression with adaptive lifting scheme using Artificial Bee Colony algorithm", International Journal of Advanced Research in Computer Engineering \& Technology (IJARCET) Volume 2, Issue 4, April 2013.

[11] I.boukli hacene, M. beladghem, A.bessaid, "Lossy Compression Color Medical Image Using CDF Wavelet Lifting Scheme", I.J. Image, Graphics and Signal Processing, 11, 53-60, September 2013.

[12] Tilak Mukherjee, M.Koteswara Rao, “ Efficient Performance of Lifting Scheme Along With Integer Wavelet Transform In Image Compression", International Journal of Engineering Research and Applications (IJERA), Vol. 3, Issue 4, Jul-Aug 2013, pp.1950-1953.

[13] M. Santhosh, Dr. B. Stephen Charles and Dr. M N Giriprasad, "Lossless Image Compression Using New Biorthogonal Wavelets", Signal \& Image Processing: An International Journal (SIPIJ) Vol.4, No.6, December 2013.

[14] G. Chenchu Krishnaiah, T. Jayachandra Prasad, M.N. Giri Prasad, “Algorithms for Improved Image Compression and Reconstruction Performances", Signal \& Image Processing: An International Journal, 2012.

[15] M. Santhosh, B. Stephen Charles and M. N. Giri Prasad, "Image Representation by First Generation Wavelets and Its Application to Compression", CiiT International Journal of Digital Image Processing, June 2013.

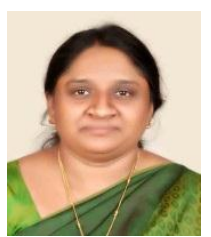

M. Santhosh, born on $1^{\text {st }}$ July 1975, received B.Tech degree from Sri Venkateswara University, Tirupati, India in 1997, M.Tech degree from Maharaj Sayaji Rao University, Baroda in 1999. She has started her career as an Asst. Prof., in AITS, Rajampet in 1999 and presently working as an Asso. Prof., in Stanley Stephen College of Engineering and Technology, Kurnool.She has more than 13 years of reacing experience. Her research interests include image representation techniques, compression and wavelets.

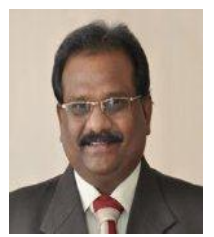

Dr. B. Stephen Charles (India) born on the 9th of August 1965. He received Ph.d degree in Electronics \& Communication Engineering from Jawaharlal Nehru Technological University, Hyderabad in 2001. His area of interest is Digital signal Processing. He received his B.Tech degree in Electronics and Communication Engineering from Nagarjuna University, India in 1986. He started his carrier as Assistant professor in Karunya institute of technology during 1989 to 1993, later joined as Associate Professor in K. S. R. M. College of Engg. During 1993 to 2001 after that he worked as Principal of St. John's College of Engineering \& Technology during 2001 to 2007 and now he is the Secretary, Correspondent and Principal in Stanley Stephen College of Engineering \& Technology, Kurnool. He has more than 25 years of teaching and research experience. He published more than 40 research papers in national and international journals and more than 30 research papers in national and international conferences. He is a member of Institute of Engineers and ISTE.

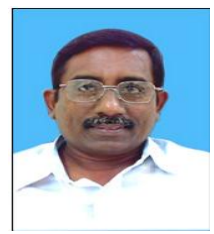

Dr. M.N. Giri Prasad received his B.Tech degree from J.N.T University College of Engineering, Anantapur, Andhrapradesh, India in 1982. M.Tech degree from Sri Venkateshwara University, Tirupati, Andhra Pradesh, India in 1994 and Ph.D degree from J.N.T. University, Hyderabad, Andhra Pradesh, Indian in 2003. Presently he is working as a Professor in the Department of Electronics and Communication at J.N.T University College of Engineering Anantapur, Andhrapradesh, India. He has more than 25 years of teaching and research experience. He has published more than 50 papers in national and international journals and more than 30 research papers in national and international conferences. His research areas are Wireless Communications and Biomedical instrumentation, digital signal processing, VHDL coding and evolutionary computing. He is a member of ISTE, IE \& NAFEN. 